\title{
Impaired Cardiac Sympathetic Innervation and Myocardial Perfusion Are Related to Lethal Arrhythmia: Quantification of Cardiac Tracers in Patients with ICDs
}

Kimio Nishisato ${ }^{1}$, Akiyoshi Hashimoto ${ }^{2}$, Tomoaki Nakata ${ }^{2,3}$, Takahiro Doi $^{2}$, Hitomi Yamamoto ${ }^{2}$ Daigo Nagahara ${ }^{4}$, Shinya Shimoshige ${ }^{2}$, Satoshi Yuda $^{2}$, Kazufumi Tsuchihashi ${ }^{2}$, and Kazuaki Shimamoto ${ }^{2}$

${ }^{I}$ Division of Cardiology, Muroram City General Hospital, Muroran, Japan; ${ }^{2}$ Sapporo Medical University School of Medicine, Sapporo, Japan; ${ }^{3}$ Hokkaido Prefectural Esashi Hospital, Esashi, Japan; and ${ }^{4}$ Obihiro-Kosei General Hospital, Obihiro, Japan

Despite widespread prophylactic use of implantable cardioverter defibrillator (ICD) therapy, sudden cardiac death and refractory arrhythmia events are still important clinical issues to be overcome. We examined whether the impairment of cardiac sympathetic innervation and myocardial perfusion is responsible for lethal arrhythmic events and has prognostic value by comparing conventional clinical indices. Methods: In consecutive ICDs implanted in 60 patients, cardiac uptake of ${ }^{123} \mathrm{I}$ metaiodobenzylguanidine and ${ }^{99 m}$ Tc-tetrofosmin at rest was quantified, and then patients were prospectively followed with endpoints of appropriate ICD shocks or cardiac death. Cardiac metaiodobenzylguanidine activity was quantified as a heart-tomediastinum ratio (HMR), and impaired tetrofosmin uptake was graded as a summed score (SS) using a computerized technique with a percentage of tracer uptake. Results: During a mean 29-mo interval, ICD shock was documented in 30 patients (50\%); 3 cardiac deaths were also observed in this group of patients. Patients with ICD shocks had a significantly smaller HMR and a greater SS than did those without (1.73 \pm 0.34 vs. $2.06 \pm 0.46, P=0.003$, and $18.0 \pm 16.2$ vs. $5.7 \pm 4.4, P=$ 0.001 , respectively). Kaplan-Meier analysis showed that patients who had both an HMR of 1.90 or less and an SS of 12 or greater had a significantly greater ICD discharge rate than did those who had both an HMR greater than 1.90 and an SS less than 12 (94\% vs. $18 \%, P<0.005)$ (log rank, 15.14; $P<$ 0.0005). Multivariate analysis with a Cox model identified the greatest Wald $\chi^{2}$ of 6.454 and a hazard ratio of $3.857(P=$ 0.011 ) when an HMR of 1.9 or less and tetrofosmin SS of 12 or greater were combined. Conclusion: Impairment of cardiac sympathetic innervation and myocardial perfusion is related to lethal arrhythmic events leading to sudden death, and the combined assessment of these can identify patients for whom prophylactic ICD use has the greatest potential.

Received Jan. 20, 2010; revision accepted Mar. 11, 2010.

For correspondence or reprints contact: Kimio Nishisato, Division of Cardiology, Muroram City General Hospital, Yamanotecho-3, Muroran 0518512, Japan.

E-mail: nishisato2008@yahoo.co.jp

COPYRIGHT ๑ 2010 by the Society of Nuclear Medicine, Inc.
Key Words: implantable cardioverter defibrillator; metaiodobenzylguanidine imaging; myocardial perfusion imaging; sudden cardiac death; lethal arrhythmias

J Nucl Med 2010; 51:1241-1249

DOI: 10.2967/jnumed.110.074971

$\mathbf{P}$ rophylactic use of implantable cardioverter defibrillators (ICDs) is established in patients with depressed left ventricular function or symptomatic heart failure at a high risk of life-threatening ventricular tachyarrhythmias $(1,2)$. Widespread use of ICD therapy, however, has increased the number of patients, medical costs, and also unfavorable effects related to this device, especially in patients at a lower risk for sudden cardiac death (3). The American College of Cardiology, American Heart Association, and North American Society for Pacing and Electrophysiology 2002 guideline for ICD implantation $(4,5)$ states that the careful selection of patients for ICD therapy is crucial from scientific, practical, and economic points of view. Invasive electrophysiologic study does not necessarily provide information on the appropriate selection of patients for ICD therapy (6), whereas history of syncope, lethal ventricular tachyarrhythmias, and recovery from cardiopulmonary resuscitation are shown to be important clinical determinants. Thus, pathophysiologically based assessment of overall heart conditions-including triggers, substrates, and modulators of fatal arrhythmic events-seems to be more reasonable for the indication of ICD therapy than is the detection of localized electrophysiologic abnormalities (7). Microcirculation at a myocyte level, myocardial viability, or cell injury seems to be principally related to arrhythmogenesis and can be assessed by myocardial perfusion tracer activity $(8,9)$. Electrophysiologic instability is an important trigger of cardiac arrhythmias and is modulated by autonomic function $(10,11)$. Cardiac sympathetic innervation and function can be assessed by quantification of ${ }^{123} \mathrm{I}-$ 
metaiodobenzylguanidine activity (12). Previous studies (12-20) have shown that alterations of cardiac metaiodobenzylguanidine kinetics closely correlate with lethal events and long-term mortality in patients with left ventricular dysfunction or heart failure. However, whether altered cardiac sympathetic innervation predicts future lethal arrhythmic events was not fully investigated. From our earlier results $(12,20)$ on the limited possibility of cardiac neuroimaging, we hypothesized that both impairment of myocardial perfusion or cell viability and sympathetic innervation are responsible for arrhythmogenesis. This study investigated prognostic implications of cardiac activities of metaiodobenzylguanidine and perfusion tracer in patients treated with prophylactic use of ICD by correlation with lethal arrhythmic events prospectively documented.

\section{MATERIALS AND METHODS}

\section{Patient Population}

This study enrolled 60 consecutive patients (mean age, 53 y; 41 men and 19 women) who had undergone ICD treatment. The presenting study protocol was based on the guidelines for ICD implantation of the American Heart Association and American College of Cardiology or on standard clinical criteria, such as survival from cardiopulmonary resuscitation or the presence of spontaneous or inducible ventricular fibrillation or refractory lifethreatening ventricular tachyarrhythmias independent of left ventricular ejection fraction (LVEF) and etiology. No patients, however, had acute ischemic events at least several months before ICD implantation. ICDs (Micro Jewel II, GEM, and GEM II [Medtronics Inc.] or VENTAK MINI IV [Guidant Inc.]) were implanted using a subclavian venous approach. Informed consent for ICD implantation and participation in this study was obtained following the guidelines of the ethics committee of our hospital. The present study population included 23 patients who had been also enrolled in our previous study (20). The clinical backgrounds, underlying cardiac diseases, and quantitative data listed in Table 1 were obtained at a stable clinical condition under appropriate medical treatment, within a few days of cardiac metaiodobenzylguanidine imaging, before discharge for a long-term follow-up at an outpatient clinic.

\section{Cardiac Sympathetic Nerve and Perfusion Imaging}

All patients underwent both cardiac metaiodobenzylguanidine and tetrofosmin myocardial perfusion imaging within $2 \mathrm{wk}$ of ICD implantation. Cardiac sympathetic nerve imaging was done using ${ }^{123}$ I-metaiodobenzylguanidine $(111 \mathrm{MBq})$ with a high specific activity (Daiichi Radioisotope Laboratories). Cardiac planar and tomographic metaiodobenzylguanidine images were obtained at fasting and resting conditions at 15-30 min (early) and $4 \mathrm{~h}$ (late) after an intravenous tracer injection, using a $\gamma$-camera equipped with a low-energy general-purpose collimator. Cardiac ${ }^{123} \mathrm{I}$-metaiodobenzylguanidine activity was quantified as a heart-to-mediastinum ratio (HMR) by manually setting a region of interest on an upper mediastinum and whole cardiac region on a planar image. Because of the elimination of background or lung activity, washout kinetics of metaiodobenzylguanidine activity from the heart were calculated using tomographic data $(12,14-20)$. This method was reported to have a high reproducibility in our previous studies.

Myocardial perfusion imaging was performed at rest using ${ }^{99 \mathrm{~m}}$ Tc-labeled tetrofosmin $(760 \mathrm{MBq})$ at $60 \mathrm{~min}$ after an intra- venous tracer injection. Electrocardiogram (ECG)-gated SPECT data were obtained for $60 \mathrm{~s}$ per projection from 60 projections during a $360^{\circ}$ rotation, with a frame rate of 8 , using a 3-head $\gamma$-camera with a low-energy, high-resolution parallel-hole collimator and a Butterworth filter (cutoff, 0.24 cycle/pixel; power factor, 8) (GCA 9300-A/DI; Toshiba). Data were stored in a $64 \times 64$ word matrix nuclear medicine computer system, and no attenuation or scatter correction was performed. LVEF, left ventricular end-diastolic volume, and left ventricular end-systolic volume were calculated from 3-dimensional gated SPECT data using a dedicated computer program, p-FAST $(21,22)$. An automated scoring system developed in our laboratory (21) calculated myocardial tetrofosmin activity as a summed score (SS) at 25 left ventricular segments on a polar map, using a relative peak count (percentage uptake) as follows: 4, less than 40\%; 3, 40\%-49\%; $2,50 \%-59 \%$; $1,60 \%-69 \%$; and $0,70 \%$ or more.

\section{Follow-up Protocol}

After discharge, all patients were followed by cardiologists at least every 3 mo for a mean of 29 mo at an outpatient pacemaker clinic of our university hospital. The primary endpoint was fatal or near-fatal cardiac events consisting of cardiac death, appropriate ICD discharge against lethal ventricular arrhythmias, life-threatening ventricular tachyarrhythmias, and recovery from cardiopulmonary resuscitation. Drug therapy for arrhythmias, background diseases, or heart failure used at the start of the study was continued for as long as no adverse effects emerged (Table 1). Amiodarone, sotalol, class I antiarrhythmic drugs, or $\beta$-blockers were used, when indicated. Diuretics, digoxin, angiotensin converting enzyme inhibitors, angiotensin receptor blockers, or $\beta$-blockers were also used for heart failure. The appropriateness of ICD shock against sustained lethal ventricular tachycardia or fibrillation was confirmed by ECG recordings or an ICD built-in memory system.

\section{Statistics}

Statistical values are shown as mean \pm SD. Mean values between 2 groups were compared using the unpaired $t$ test, and prevalence was compared using a $\chi^{2}$ test. A $P$ value of less than 0.05 was considered significant. Receiver-operating-characteristic analysis was performed to determine optimal cutoff values of HMR and SS for primary endpoints (Fig. 1). Survival curves of a subgroup of patients were created using the Kaplan-Meier method to clarify the time-dependent cumulative event-free rate and were compared using the log-rank test. Univariate and multivariate analyses with a Cox proportional hazards model were performed using a computer statistical program package (SPSS, version 11.0; SPSS Inc.).

\section{RESULTS}

\section{Clinical Backgrounds}

During a follow-up period, ICD discharges were documented in 30 patients $(50 \%)$, in whom 3 cardiac deaths due to congestive heart failure were observed. No patients had acute myocardial infarction, acute coronary syndrome, or recovery from cardiopulmonary resuscitation during this follow-up period. There was no significant difference in age, sex, underlying cardiac disease, New York Heart Association class, or follow-up period 
TABLE 1. Comparison of Clinical Backgrounds Between Patients With and Without Primary Endpoints

\begin{tabular}{|c|c|c|c|}
\hline Parameter & $\begin{array}{l}\text { Patients with cardiac } \\
\text { events }(n=30)\end{array}$ & $\begin{array}{l}\text { Patients without cardiac } \\
\text { events }(n=30)\end{array}$ & $P$ \\
\hline Mean age $\pm S D(y)$ & $53.3 \pm 15.0$ & $53.4 \pm 12.1$ & NS \\
\hline Sex (female) & $8(27)$ & $11(37)$ & NS \\
\hline \multicolumn{4}{|l|}{ Coronary risk factors } \\
\hline Diabetes mellitus & $8(27)$ & $4(13)$ & NS \\
\hline Hypertension & $7(23)$ & $8(27)$ & NS \\
\hline Dyslipidemia & $14(47)$ & $7(23)$ & NS \\
\hline Chronic renal failure & $2(7)$ & $3(10)$ & NS \\
\hline \multicolumn{4}{|l|}{ Underlying cardiac disease } \\
\hline Prior myocardial infarction & $8(27)$ & $3(10)$ & \\
\hline Dilated cardiomyopathy & $15(50)$ & 7 (23) & \\
\hline Hypertrophic cardiomyopathy & $2(7)$ & $5(17)$ & NS \\
\hline Arrhythmogenic right ventricular dysplasia & $2(7)$ & $4(13)$ & \\
\hline Brugada syndrome & $1(3)$ & $9(30)$ & \\
\hline Idiopathic ventricular arrhythmia & $2(7)$ & $2(7)$ & \\
\hline NYHA functional class & & & NS \\
\hline 1 & 15 & 25 & \\
\hline II & 0 & 3 & \\
\hline III & 14 & 0 & \\
\hline IV & 1 & 2 & \\
\hline Mean follow-up period \pm SD (mo) & $31 \pm 17$ & $28 \pm 15$ & NS \\
\hline \multicolumn{4}{|l|}{$\mathrm{ECG}($ mean $\pm \mathrm{SD})$} \\
\hline LVEF (\%) & $42 \pm 18$ & $56 \pm 15$ & 0.002 \\
\hline Left ventricular end-diastolic diameter (mm) & $57 \pm 11$ & $50 \pm 11$ & 0.017 \\
\hline $\mathrm{BNP}(\mathrm{pg} / \mathrm{mL})$ & $209 \pm 198$ & $101 \pm 140$ & 0.018 \\
\hline \multicolumn{4}{|l|}{ Presenting arrhythmia before ICD implantation } \\
\hline VF & $6(20)$ & $7(23)$ & NS \\
\hline Sustained VT & $18(60)$ & $8(27)$ & 0.02 \\
\hline Nonsustained VT & $6(20)$ & $5(17)$ & NS \\
\hline \multicolumn{4}{|l|}{ Electrophysiologic study } \\
\hline Inducible VT & $17(57)$ & $12(40)$ & NS \\
\hline Inducible VF & $16(53)$ & $10(33)$ & NS \\
\hline \multicolumn{4}{|l|}{ Concomitant medication } \\
\hline Diuretic & $14(47)$ & $6(20)$ & NS \\
\hline Spironolactone & $15(50)$ & $4(13)$ & 0.005 \\
\hline Digitalis & $3(10)$ & $0(0)$ & NS \\
\hline$\beta$-blocker & $22(73)$ & $11(37)$ & 0.011 \\
\hline ACEI/ARB & $19(63)$ & $9(30)$ & 0.02 \\
\hline Nitrate & $6(20)$ & $1(3)$ & NS \\
\hline
\end{tabular}

NS = not significant; NYHA = New York Heart Association; VF = ventricular fibrillation; VT = ventricular tachyarrhythmias; ACEI = angiotensin converting enzyme inhibitor; ARB = angiotensin II receptor blocker.

Data in parentheses are percentages.

between patients with and without events (Table 1). Patients with cardiac events had a significantly lower $\operatorname{LVEF}(42 \% \pm 18 \%$ vs. $56 \% \pm 15 \%, P=0.002)$, a significantly larger left ventricular end-diastolic diameter (57 \pm 11 vs. $50 \pm 11 \mathrm{~mm}, P=0.017)$, and a significantly greater brain natriuretic peptide (BNP) level $(209 \pm 198$ vs. $101 \pm 140, P=0.018)$ than did those without. Patients with lethal events also had a significantly greater prevalence of sustained ventricular tachycardia (60\% vs. $27 \%, P=0.0172$ ) than did those without. However, for the electrophysiologic study there was no significant difference in prevalence of ventricular fibrillation or nonsustained ventricular tachycardia and in inducibility of lethal tachyarrhythmias between the groups. Patients with cardiac events received spironolactone, $\beta$-blockers, angiotensin converting enzyme inhibitors or angiotensin II receptor blockers, and class III antiarrhythmic drugs more frequently than did those without.

\section{Cardiac Metaiodobenzylguanidine and Gated SPECT Data}

Patients with lethal events had significantly reduced cardiac metaiodobenzylguanidine activities, compared with those without $(1.96 \pm 0.26$ vs. $2.16 \pm 0.37, P=0.019$, for early HMR and $1.73 \pm 0.34$ vs. $2.06 \pm 0.46, P=0.003$, for late HMR [Table 2]). However, there was no significant difference in metaiodobenzylguanidine washout rate between the groups. The event group had a significantly greater SS, a significantly smaller LVEF, and significantly 
FIGURE 1. Receiver-operating-characteristic analysis using cardiac metaiodobenzylguanidine activity (late HMR) and tetrofosmin SS for identification of patients with primary cardiac event. Metaiodobenzylguanidine HMR of 1.90 and tetrofosmin SS of 12 were selected as optimal cutoff values. TF $=$ tetrofos$\min$.
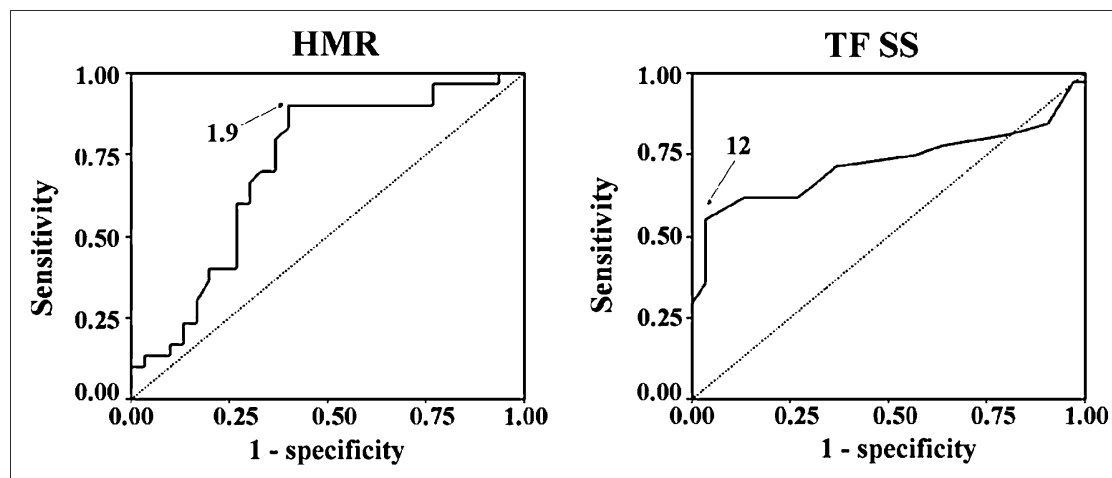

larger left ventricular volumes than did the other groups: $18.0 \pm 16.2$ vs. $5.7 \pm 4.4, P=0.001$, for SS; $36 \% \pm 19 \%$ vs. $54 \% \pm 13 \%, P=0.001$, for LVEF; $187 \pm 117 \mathrm{~mL}$ vs. $109 \pm 87 \mathrm{~mL}, P=0.005$, for left ventricular end-diastolic volume; and $157 \pm 121 \mathrm{~mL}$ vs. $75 \pm 74 \mathrm{~mL}, P=0.002$, for left ventricular end-systolic volume.

\section{Event Rates and Cardiac Uptake of Metaiodobenzylguanidine and Tetrofosmin}

Receiver-operating-characteristic analysis was performed using cardiac metaiodobenzylguanidine activity (late HMR) and myocardial perfusion abnormality (tetrofosmin SS); analysis also determined optimal cutoff values for the identification of a high-risk group (1.90 for a late HMR of cardiac metaiodobenzylguanidine activity and 12 for tetrofosmin SS) (Fig. 1). Two thresholds were used to divide the patients into the following 3 groups: patients with a late HMR of 1.90 or more and an SS less than 12 (preserved metaiodobenzylguanidine activity and tetrofosmin uptake), patients with a late HMR less than 1.90 and an SS less than 12 (reduced metaiodobenzylguanidine activity but preserved tetrofosmin uptake), and patients with a late HMR less than 1.90 and an SS of 12 or more (reduced metaiodobenzylguanidine activity and tetrofosmin uptake). There was no patient with an HMR of 1.90 or more and a SS of 12 or more. The group with impaired uptake of metaiodobenzylguanidine and tetrofosmin had a significantly greater event rate (94\%) than did the other 2 groups (45\% for the group with impaired uptake of metaiodobenzylguanidine and preserved uptake of tetrofosmin $[P<0.05]$ and $18 \%$ for the group with preserved uptake of both metaiodobenzylguanidine and tetrofosmin $[P<0.005]$ ) (Fig. 2). In addition, the group with impaired uptake of metaiodobenzylguanidine and tetrofosmin had a significantly lower event-free rate than did the group with impaired metaiodobenzylguanidine and preserved tetrofosmin uptake (log rank, $6.14 ; P=0.0132$ ) or the group with preserved uptake of both metaiodobenzylguanidine and tetrofosmin (log rank, 15.14; $P=0.0001$ ) (Fig. 3).

\section{Event-Rate Analysis in Patients with Preserved Cardiac Function}

Because 31 of 60 patients had an LVEF of $50 \%$ or more, event-rate analysis was performed only in this patient population. The group with impaired uptake of both metaiodobenzylguanidine and tetrofosmin had a significantly $(P<0.05)$ higher event rate $(100 \%)$ than did the other 2 groups $(30 \%$ for the group with impaired metaiodobenzylguanidine activity and preserved tetrofosmin uptake and $16 \%$ for the group with preserved uptake of both metaiodobenzylguanidine and tetrofosmin) (Fig. 4). Likewise, the group with impaired uptake of both metaiodobenzylguanidine and tetrofosmin had the lowest event-free rate among the 3 groups (log rank, 6.06; $P=0.0138$ ) (Fig. 5).

\section{TABLE 2. Comparison of Scintigraphic Data Between Patients With and Without Primary Endpoints}

\begin{tabular}{|c|c|c|c|}
\hline Parameter & Patients with cardiac events $(n=30)$ & Patients without cardiac events $(n=30)$ & $P$ \\
\hline \multicolumn{4}{|c|}{ Metaiodobenzylguanidine } \\
\hline Early HMR & $1.96 \pm 0.26$ & $2.16 \pm 0.37$ & 0.019 \\
\hline Late HMR & $1.73 \pm 0.34$ & $2.06 \pm 0.46$ & 0.003 \\
\hline Washout rate & $33.1 \pm 14.0$ & $28.6 \pm 11.0$ & NS \\
\hline \multicolumn{4}{|l|}{ Tetrofosmin } \\
\hline SS & $18.0 \pm 16.2$ & $5.7 \pm 4.4$ & 0.000 \\
\hline LVEF & $36 \pm 19$ & $54 \pm 13$ & 0.000 \\
\hline LVEDV & $187 \pm 117$ & $109 \pm 87$ & 0.005 \\
\hline LVESV & $157 \pm 121$ & $75 \pm 74$ & 0.002 \\
\hline
\end{tabular}




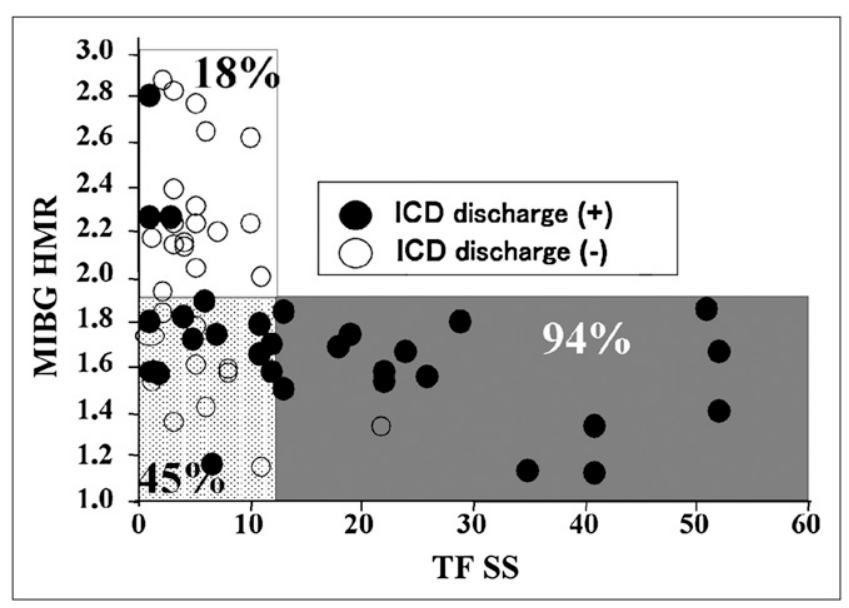

FIGURE 2. Scatterplots of patients with $(\bullet)$ and without $(\bigcirc)$ primary cardiac events who were divided into 3 groups based on cutoff vales of cardiac metaiodobenzylguanidine activity and tetrofosmin uptake identified by receiveroperating-characteristic analysis. There are significant $(P<$ $0.05)$ differences in event rate among the 3 groups $(94 \%$ for group with impaired metaiodobenzylguanidine and tetrofosmin uptake, $45 \%$ for group with impaired metaiodobenzylguanidine and preserved tetrofosmin uptake, and $18 \%$ for group with preserved metaiodobenzylguanidine and tetrofosmin uptake). Unshaded area $=$ preserved uptake of both metaiodobenzylguanidine and tetrofosmin $(n=22)$; dotted area $=$ impaired metaiodobenzylguanidine uptake and preserved tetrofosmin uptake $(n=20)$; shaded area = impaired uptake of both metaiodobenzylguanidine and tetrofosmin $(n=18)$. MIBG $=$ metaiodobenzylguanidine; $\mathrm{TF}=$ tetrofosmin.

\section{Univariate and Multivariate Analyses}

BNP level, a metaiodobenzylguanidine HMR of 1.9 or less, a tetrofosmin SS of 12 or more, and LVEF were chosen for further multivariate analysis with a Cox model, on the basis of the univariate analysis results (Table 3 ) in

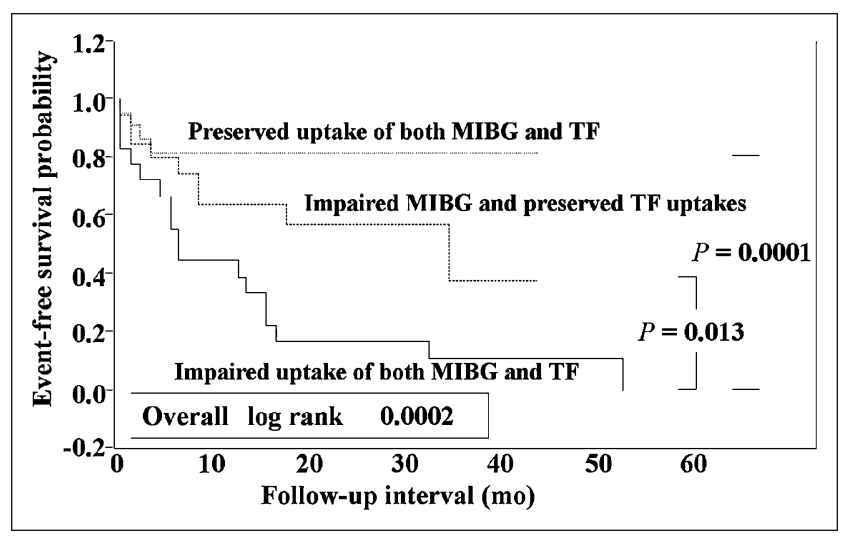

FIGURE 3. Kaplan-Meier event-free curves of 3 groups based on metaiodobenzylguanidine and tetrofosmin uptake show that patients with impaired uptake of both metaiodobenzylguanidine and tetrofosmin had significantly lower event-free rate than did patients in the other 2 groups. $\mathrm{MIBG}=$ metaiodobenzylguanidine; TF $=$ tetrofosmin. combination with the cutoff values of HMR and tetrofosmin SS (Fig. 1). The greatest Wald $\chi^{2}$ of 6.454 and a hazard ratio of $3.857(P=0.011)$ were identified when an HMR of 1.9 or less and a tetrofosmin SS of 12 or more were combined (Table 3).

\section{Patient Presentations}

Figure 6 shows planar metaiodobenzylguanidine and tetrofosmin images and SPECT images of 2 typical patients with and without lethal arrhythmic events. The patient who had markedly reduced metaiodobenzylguanidine activity and profound perfusion abnormality had several ICD shocks against an electrical storm of ventricular tachyarrhythmias 3 mo after ICD implantation, whereas the patient with preservation of metaiodobenzylguanidine activity and myocardial perfusion had neither ICD shock nor other cardiac events for 30 mo.

\section{DISCUSSION}

The present results clearly demonstrated the incremental benefits of combined assessment of myocardial perfusion and sympathetic nerve function for appropriate risk stratification of patients who are likely to need prophylactic ICD therapy.

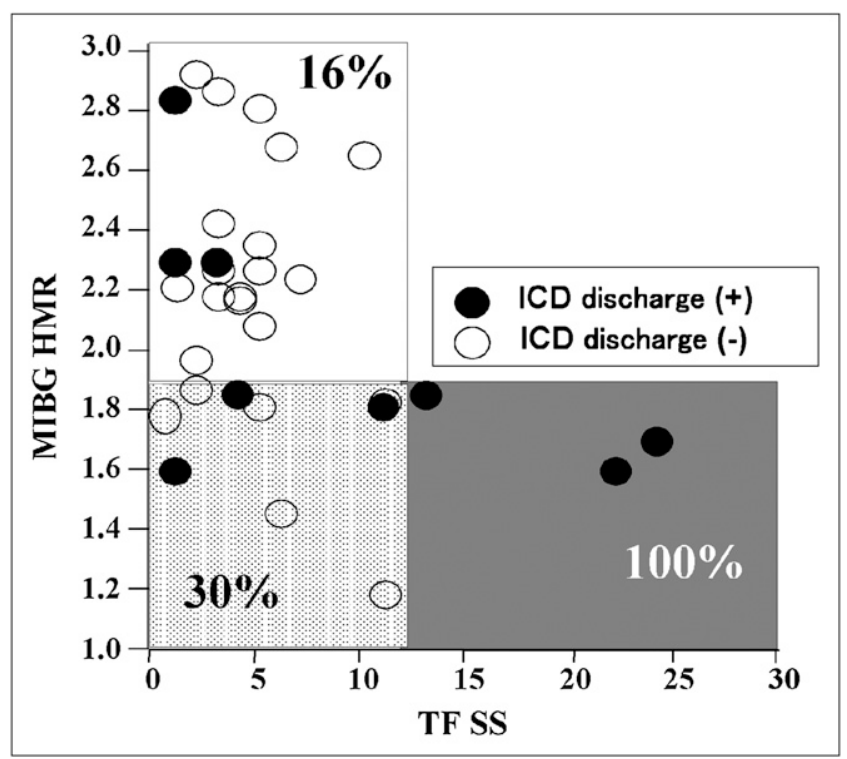

FIGURE 4. Scatterplots of patients with (O) and without (O) primary cardiac events when 31 patients with LVEF of $50 \%$ or more are considered. Patient group with impaired uptake of both metaiodobenzylguanidine and tetrofosmin had greatest $(P<0.05)$ event rate $(100 \%)$ when compared with other groups $(16 \%-30 \%)$. Unshaded area = preserved uptake of both metaiodobenzylguanidine and tetrofosmin $(n=19)$; dotted area = impaired metaiodobenzylguanidine uptake and preserved tetrofosmin uptake $(n=9)$; shaded area $=$ impaired uptake of both metaiodobenzylguanidine and tetrofosmin $(n=3)$. MIBG = metaiodobenzylguanidine; $\mathrm{TF}=$ tetrofosmin 


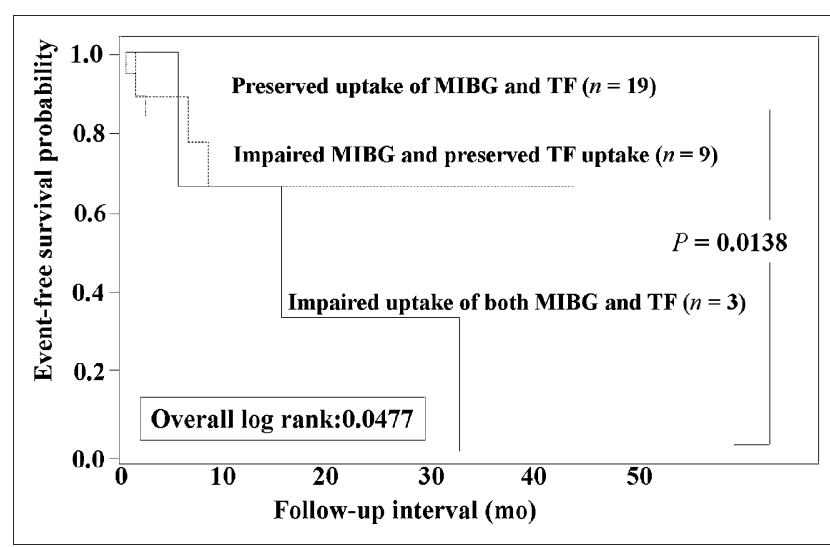

FIGURE 5. Kaplan-Meier event-free curves of 3 groups based on metaiodobenzylguanidine and tetrofosmin uptake when 31 patients with LVEF of $50 \%$ or more are considered. Patients with impaired uptake of both metaiodobenzylguanidine and tetrofosmin had significantly lower event-free rate than did group with preserved uptake of both metaiodobenzylguanidine and tetrofosmin. MIBG = metaiodobenzylguanidine; TF $=$ tetrofosmin .

\section{Myocardial Perfusion Assessment} and Arrhythmogenicity

Organic tissue injury or sustained perfusion abnormality is responsible for fatal arrhythmic events, probably because of the function of substrate for arrhythmogenicity such as reentrant circuit and abnormal automaticity $(23,24)$. A decrease in myocardial perfusion tracer uptake at rest indicates sustained impairment of microcirculation, organic tissue injury, or adenosine triphosphate depletion state (10).
These findings support the rationale for the assessment of myocardial perfusion tracer uptake in patients at lethal arrhythmic event risk. The present study quantified myocardial activity of ${ }^{99 \mathrm{~m}} \mathrm{Tc}$-labeled tetrofosmin to evaluate myocardial perfusion abnormality, viability, and tissue injury using the computerized automated scoring system with a polar-map technique $(21,22)$.

\section{Assessment of Cardiac Sympathetic Innervation and Arrhythmogenicity}

Lethal arrhythmias occur at the end of pathophysiologic cascades because of complex interactions between structural injury and altered autonomic function (25). Alterations of autonomic tones, electrolyte imbalance, and hormonal factors are responsible for arrhythmogenesis $(23,26)$. A recent preliminary study (27) suggested the arrhythmogenicity of regional metaiodobenzylguanidine defect. Compared with invasive testing $(20,28)$, however, signal-averaged ECG, T-wave alternans, QT dispersion, and heart rate variability are noted as more sensitive markers (29-32), because electrophysiologic inducibility of ventricular tachyarrhythmias is not necessarily related to clinically documented arrhythmic events or outcomes. Although appropriate ICD shocks most likely occur when patients have both reduced cardiac metaiodobenzylguanidine activity and depressed heart rate variability (12), electrophysiologic indices vary over short intervals, dependent on artifacts, rhythm disturbance, and noncardiac autonomic tone (33). In our previous study (20), electrophysiologic inducibility of lethal ventricular tachyarrhythmias and signal-averaged ECG indices, when compared with cardiac metaiodobenzylguanidine activity, were not independent

TABLE 3. Univariate and Multivariate Logic Regression Analyses

\begin{tabular}{|c|c|c|c|c|c|c|c|c|c|c|}
\hline \multirow[b]{3}{*}{ Parameter } & \multicolumn{5}{|c|}{ Univariate } & \multicolumn{5}{|c|}{$\begin{array}{c}\text { Multivariate } \\
\text { (Cox proportional hazards model) }\end{array}$} \\
\hline & \multirow[b]{2}{*}{ Wald } & \multirow[b]{2}{*}{ Hazard ratio } & \multicolumn{2}{|c|}{$\begin{array}{c}95 \% \\
\text { Confidence } \\
\text { interval }\end{array}$} & \multirow[b]{2}{*}{$P$} & \multirow[b]{2}{*}{ Wald } & \multirow[b]{2}{*}{ Hazard ratio } & \multicolumn{2}{|c|}{$\begin{array}{c}95 \% \\
\text { Confidence } \\
\text { interval }\end{array}$} & \multirow[b]{2}{*}{$P$} \\
\hline & & & Lower & Upper & & & & Lower & Upper & \\
\hline Age $(y)$ & 0.050 & 0.997 & 0.969 & 1.026 & 0.824 & & & & & \\
\hline Sex & 0.908 & 1.489 & 0.657 & 3.376 & 0.341 & & & & & \\
\hline Clinical VT/VF & 3.992 & 4.34 & 1.028 & 18.313 & 0.046 & & & & & \\
\hline Inducible VT/VF & 0.210 & 0.839 & 0.396 & 1.779 & 0.647 & & & & & \\
\hline SAECG & 0.574 & 1.492 & 0.530 & 4.198 & 0.449 & & & & & \\
\hline Medications & 1.615 & 1.629 & 0.767 & 3.459 & 0.204 & & & & & \\
\hline BNP & 4.579 & 1.002 & 1.000 & 1.004 & 0.032 & 0.655 & 1.001 & 0.998 & 1.004 & 0.418 \\
\hline HMR (late) & 7.771 & 0.205 & 0.067 & 0.625 & 0.005 & & & & & \\
\hline Washout & 0.663 & 1.014 & 0.981 & 1.047 & 0.415 & & & & & \\
\hline $\mathrm{HMR} \leqq 1.9$ & 8.509 & 5.966 & 1.797 & 19.806 & 0.004 & 3.705 & 4.560 & 0.973 & 21.374 & 0.054 \\
\hline Tetrofosmin SS & 13.547 & 1.043 & 1.020 & 1.067 & 0.000 & & & & & \\
\hline Tetrofosmin SS $\geqq 12$ & 13.691 & 4.022 & 1.924 & 8.408 & 0.000 & 4.083 & 2.842 & 1.032 & 7.830 & 0.043 \\
\hline $\begin{array}{l}\mathrm{HMR} \leqq 1.9 \text { and } \\
\text { tetrofosmin } \mathrm{SS} \geqq 12\end{array}$ & 13.691 & 4.022 & 1.924 & 8.408 & 0.000 & 6.454 & 3.857 & 1.361 & 10.928 & 0.011 \\
\hline LVEF & 4.188 & 0.978 & 0.958 & 0.999 & 0.041 & 0.890 & 0.346 & 0.983 & 1.051 & 0.346 \\
\hline
\end{tabular}




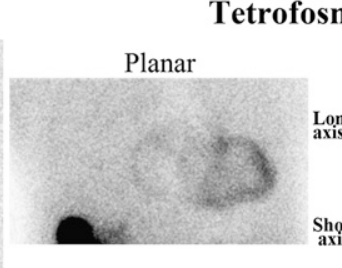

$\mathrm{HMR}=1.21$

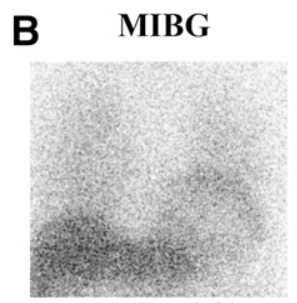

$\mathrm{HMR}=\mathbf{2 . 0 2}$

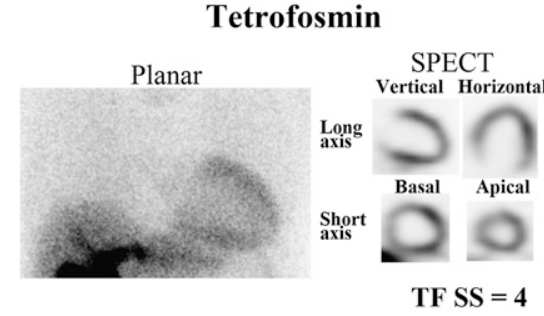

FIGURE 6. Planar metaiodobenzylguanidine images and planar and tomographic tetrofosmin images. (A) A 56-y-old woman had both markedly reduced metaiodobenzylguanidine activity (HMR of 1.21) and perfusion uptake (tetrofosmin SPECT SS of 16) and underwent ICD shocks 3 mo after implantation because of electrical storm of ventricular tachyarrhythmia. (B) A 60-y-old man had highly dilated left ventricle but nearly normal cardiac metaiodobenzylguanidine activity (HMR of 2.02) and perfusion score (tetrofosmin SS of 4). Neither ICD shock nor other lethal cardiac event was observed during follow-up. MIBG = metaiodobenzylguanidine; TF = tetrofosmin.

significant predictors of lethal arrhythmic events. In this study, metaiodobenzylguanidine washout rate was not a dominant predictor of events. Metaiodobenzylguanidine washout rate is a functional marker of cardiac sympathetic tone when an initial uptake of metaiodobenzylguanidine is preserved to some extent and when extraneuronal components are excluded. Washout rate is, however, overestimated significantly when cardiac metaiodobenzylguanidine uptake is globally depressed, as often observed in patients with extensive myocardial injury or severe heart failure. In contrast, late HMR is determined both by an initial metaiodobenzylguanidine uptake (sympathetic innervation and uptake-1 system) and by washout rate, which is regulated by central stimulation, norepinephrine release, reuptake, and spillover. Thus, late HMR can express overall cardiac presynaptic innervation and function, making this method a more reliable prognostic marker than washout rate.

\section{Risk Stratification Using Metaiodobenzylguanidine} Activity in Combination with Myocardial Perfusion

Postinfarction sympathetic denervation assessed by cardiac metaiodobenzylguanidine activity is shown to be arrhythmogenic (24); in addition, experimentally denervated but viable myocardium is supersensitive to catecholamine and arrhythmogenesis because of electrophysiologic instability (34). These findings support the rationale for the combined use of metaiodobenzylguanidine and perfusion tracer for the improvement of risk stratification in patients at lethal arrhythmic event risk, for the appropriate selection of prophylactic ICD candidates and prediction of efficacy of ICD treatment.

Left ventricular systolic dysfunction is a powerful predictor of major cardiac events in coronary patients and in heart failure patients but is not necessarily related to lethal arrhythmic events $(35,36)$. Long-term benefits of ICD are shown to be limited independent of $\operatorname{LVEF}(37,38)$. Similarly, this study demonstrated that the imaging technique effectively risk-stratified patients independently of LVEF. In contrast to our previous findings $(14,15,18,20)$, plasma BNP level and LVEF were unlikely to provide additional benefits to prognosis assessment by cardiac metaiodobenzylguanidine or perfusion technique. There are several possible explanations. First, LVEF and New York Heart Association functional class were relatively well preserved; second, the endpoint was lethal arrhythmic events but not limited to cardiac death alone; third, impaired diastolic performance, which is often overlooked in heart failure patients, may be related to lethal arrhythmic events; and finally, cardiac metaiodobenzylguanidine activity had substantially powerful prognostic values, particularly in combination with myocardial perfusion assessment in this study population. Thus, myocardial tissue injury and altered autonomic innervation identified by perfusion tracer and metaiodobenzylguanidine activities are closely and complementarily related to unfavorable outcomes.

\section{Study Limitations}

This study assessed global, but not regional, cardiac metaiodobenzylguanidine activity and did not evaluate autonomic heterogeneity or localization of arrhythmogenicity. When cardiac metaiodobenzylguanidine activity is regionally, but not globally, impaired-as seen in patients with coronary artery disease without severe heart failureregional SPECT analysis is possible (17,27). It is, however, difficult to reconstruct SPECT data (27) in the case of globally defective metaiodobenzylguanidine uptake, which is often seen in patients with severe heart failure or left ventricular dysfunction. Furthermore, the lower the global metaiodobenzylguanidine activity is, the poorer a patient's prognosis becomes (18). It is also known that cardiac metaiodobenzylguanidine uptake is underestimated at inferior and apical walls without definitive myocardial damage, probably for attenuation and physiologic reasons $(17,39)$. Although this technical problem lowers the efficacy of SPECT for prognostic assessment, precise segment-to-segment metaiodobenzylguanidine analysis using a high-quality SPECT image may be able to provide information for prognosis and globally assessed cardiac metaiodobenzylguanidine activity $(27,40)$. Because this study focused on lethal events under ICD treatment in cardiology practice, patients were consecutively enrolled to reduce selection bias. As a result, patient backgrounds varied and the heterogeneity may have affected the presented results. Despite 
these limitations, the presented results revealed prognostic efficacies of the assessment of both cardiac sympathetic innervation and myocardial perfusion and suggest the possibility of this method for determining ICD indication in real-world clinical settings, independently of various clinical backgrounds. A cost-benefit analysis is also an important practical issue. If the nuclear techniques are used for appropriate selection of ICD candidates, not only the overuse but also the underuse of ICD could be avoided and the assessment could be more cost-effective. Cardiac metaiodobenzylguanidine activity might alter during clinical course observation, depending on disease process or treatments. It remains to be clarified when and how long cardiac metaiodobenzylguanidine activity should be followed up in patients at high risk for sudden cardiac death. Finally, the presented results emphasize the need for a multicenter study with a larger sample size and a longer follow-up interval for establishing the clinical implications of the presented technique.

\section{CONCLUSION}

Impaired cardiac sympathetic innervation and myocardial perfusion abnormality are related to lethal arrhythmic events leading to sudden death. Thus, the combined imaging techniques using a neurotransmitter analog and a perfusion tracer can provide crucial information not only on myocardial arrhythmogenesis but also on the selection of patients at greater risk for sudden death, for most reasonable candidates of prophylactic ICD use.

\section{REFERENCES}

1. Bristow MR, Saxon LA, Boehmer J, et al. Comparison of Medical Therapy, Pacing, and Defibrillation in Heart Failure (COMPANION) Investigators. Cardiac-resynchronization therapy with or without an implantable defibrillator in advanced chronic heart failure. N Engl J Med. 2004;350:2140-2150.

2. Knight BP, Goyal R, Pelosi F, et al. Outcome of patients with nonischemic dilated cardiomyopathy and unexplained syncope treated with an implantable defibrillator. J Am Coll Cardiol. 1999;33:1964-1970.

3. Randomized antiarrhythmic drug therapy in survivors of cardiac arrest (the CASCADE Study). The CASCADE Investigators. Am J Cardiol. 1993;72: 280-287.

4. Kadish A, Dyer A, Daubert JP, et al. Defibrillators in Non-Ischemic Cardiomyopathy Treatment Evaluation (DEFINITE) Investigators. Prophylactic defibrillator implantation in patients with nonischemic dilated cardiomyopathy. $N$ Engl J Med. 2004;350:2151-2158.

5. Gregoratos G, Abrams J, Epstein AE, et al. ACC/AHA/NASPE 2002 guideline update for implantation of cardiac pacemakers and antiarrhythmia devices: summary article-a report of the American College of Cardiology/American Heart Association Task Force on Practice Guidelines (ACC/AHA/NASPE Committee to Update the 1998 Pacemaker Guidelines). Circulation. 2002;106: 2145-2161.

6. Sweeney MO, Ruskin JN. Mortality benefits and the implantable cardioverterdefibrillator. Circulation. 1994;89:1851-1858.

7. Sanders GD, Hlatky MA, Owens DK. Cost-effectiveness of implantable cardioverter-defibrillators. $N$ Engl J Med. 2005;353:1471-1480.

8. Eguchi M, Tsuchihashi K, Hotta D, et al. Technetium-99m sestamibi/tetrofosmin myocardial perfusion scanning in cardiac and noncardiac sarcoidosis. Cardiology. 2000;94:193-199.

9. Eguchi M, Tsuchihashi K, Nakata T, Hashimoto A, Shimamoto K. Right ventricular abnormalities assessed by myocardial single-photon emission computed tomography using technetium-99m sestamibi/tetrofosmin in right ventricle-originated ventricular tachyarrhythmias. J Am Coll Cardiol. 2000;36: 1767-1773.

10. Gradel C, Jain D, Batsford WP, Wackers FJ, Zaret BL. Relationship of scar and ischemia to the results of programmed electrophysiological stimulation in patients with coronary artery disease. J Nucl Cardiol. 1997;4:379-386.

11. Wichter $T$, Hindricks $G$, Lerch $H$, et al. Regional myocardial sympathetic dysinnervation in arrhythmogenic right ventricular cardiomyopathy: an analysis using ${ }^{123}$ I-meta-iodobenzylguanidine scintigraphy. Circulation. 1994;89:667-683.

12. Arora R, Ferrick KJ, Nakata T, et al. I-123 MIBG imaging and heart rate variability analysis to predict the need for an implantable cardioverter defibrillator. J Nucl Cardiol. 2003;10:121-131.

13. Nakata T, Wakabayashi T, Kyuma M, Takahashi $T$, Tsuchihashi $K$, Shimamoto K. Cardiac metaiodobenzylguanidine activity can predict the longterm efficacy of angiotensin-converting enzyme inhibitors and/or betaadrenoceptor blockers in patients with heart failure. Eur J Nucl Med Mol Imaging. 2005;32:186-194.

14. Kyuma M, Nakata T, Hashimoto A, et al. Incremental prognostic implications of brain natriuretic peptide, cardiac sympathetic nerve innervation, and noncardiac disorders in patients with heart failure. J Nucl Med. 2004;45:155-163.

15. Wakabayashi T, Nakata T, Hashimoto A, et al. Assessment of underlying etiology and cardiac sympathetic innervation to identify patients at high risk of cardiac death. J Nucl Med. 2001;42:1757-1767.

16. Nakata T, Wakabayashi T, Kyuma M, et al. Prognostic implications of an initial loss of cardiac metaiodobenzylguanidine uptake and diabetes mellitus in patients with left ventricular dysfunction. J Card Fail. 2003;9:113-121.

17. Nakata T, Nagao K, Tsuchihashi K, Hashimoto A, Tanaka S, Iimura O. Regional cardiac sympathetic nerve dysfunction and the diagnostic efficacy of metaiodobenzylguanidine tomography in stable coronary artery disease. Am J Cardiol. 1996;78:292-297.

18. Nakata T, Miyamoto K, Doi A, et al. Cardiac death prediction and impaired cardiac sympathetic innervation assessed by MIBG in patients with failing and nonfailing hearts. J Nucl Cardiol. 1998;5:579-590.

19. Nakata T, Shimamoto K, Yonekura S, et al. Cardiac sympathetic denervation in transthyretin-related familial amyloidotic polyneuropathy: detection with iodine123-MIBG. J Nucl Med. 1995;36:1040-1042.

20. Nagahara D, Nakata T, Hashimoto A, et al. Predicting the need for an implantable cardioverter defibrillator using cardiac metaiodobenzylguanidine activity together with plasma natriuretic peptide concentration or left ventricular function. J Nucl Med. 2008;49:225-233.

21. Nakata T, Katagiri Y, Odawara Y, et al. Two- and three-dimensional assessments of myocardial perfusion and function by using technetium- $99 \mathrm{~m}$ sestamibi gated SPECT with a combination of count- and image-based techniques. $J$ Nucl Cardiol. 2000;7:623-632.

22. Hashimoto A, Nakata T, Wakabayashi $T$, et al. Validation of quantitative gated SPECT and an automated scoring system for the assessment of regional left ventricular systolic function. Nucl Med Commun. 2002;23:887-898.

23. Myerburg RJ, Kessler KM, Bassett AL, Castellanos A. A biological approach to sudden cardiac death: structure, function, and cause. Am J Cardiol. 1989;63:1512-1516.

24. Matsunari I, Schricke U, Bengel FM, et al. Extent of cardiac sympathetic neuronal damage is determined by the area of ischemia in patients with acute coronary syndromes. Circulation. 2000;101:2579-2585.

25. Kjekshus J. Arrhythmia and mortality in congestive heart failure. Am J Cardiol. 1990;65:42I-48I.

26. Willich SN, Maclure M, Mittleman M, Arntz HR, Muller JE. Sudden cardiac death: support for a role of triggering in causation. Circulation. 1993;87:1442-1450.

27. Bax JJ, Kraft O, Buxton AE, et al. ${ }^{123} \mathrm{I}-m \mathrm{IBG}$ scintigraphy to predict inducibility of ventricular arrhythmias on cardiac electrophysiology testing: a prospective multicenter pilot study. Circ Cardiovasc Imaging. 2008;1: 131-140.

28. Maisel WH, Sweeney MO, Stevenson WG, Ellison KE, Epstein LM. Recalls and safety alerts involving pacemakers and implantable cardioverter-defibrillator generators. JAMA. 2001;286:793-799.

29. Gomes JA, Cain ME, Buxton AE, Josephson ME, Lee KL, Hafley GE. Prediction of long-term outcomes by signal-averaged electrocardiography in patients with unsustained ventricular tachycardia, coronary artery disease, and left ventricular dysfunction. Circulation. 2001;104:436-441.

30. Bloomfield DM, Steinman RC, Namerow PB, et al. Microvolt T-wave alternans distinguishes between patients likely and patients not likely to benefit from implanted cardiac defibrillator therapy: a solution to the Multicenter Automatic Defibrillator Implantation Trial (MADIT) II conundrum. Circulation. 2004;110:1885-1889. 
31. Brendorp B, Elming H, Jun L, et al. QT dispersion has no prognostic information for patients with advanced congestive heart failure and reduced left ventricular systolic function. Circulation. 2001;103:831-835.

32. La Rovere MT, Pinna GD, Maestri R, et al. Short-term heart rate variability strongly predicts sudden cardiac death in chronic heart failure patients. Circulation. 2003;107:565-570.

33. Shusterman V, Goldberg A, London B. Upsurge in T-wave alternans and nonalternating repolarization instability precedes spontaneous initiation of ventricular tachyarrhythmias in humans. Circulation. 2006;113:2880-2887.

34. Inoue H, Zipes DP. Result of sympathetic denervation in the canine heart: supersensitivity that may be arrhythmogenic. Circulation. 1987;75:877-887.

35. Romeo F, Pelliccia F, Cianfrocca C, Cristofani R, Reale A. Predictors of sudden death in idiopathic dilated cardiomyopathy. Am J Cardiol. 1989;63: $138-140$.

36. Bhatia RS, Tu JV, Lee DS, et al. Outcome of heart failure with preserved ejection fraction in a population-based study. N Engl J Med. 2006;355:260-269.
37. Böcker D, Bänsch D, Heinecke A, et al. Potential benefit from implantable cardioverter-defibrillator therapy in patients with and without heart failure. Circulation. 1998;98:1636-1643.

38. Goldberger JJ, Cain ME, Hohnloser SH, et al. American Heart Association/ American College of Cardiology Foundation/Heart Rhythm Society scientific statement on noninvasive risk stratification techniques for identifying patients at risk for sudden cardiac death: a scientific statement from the American Heart Association Council on Clinical Cardiology Committee on Electrocardiography and Arrhythmias and Council on Epidemiology and Prevention. J Am Coll Cardiol. 2008;52:1179-1199.

39. Tsuchimochi S, Tamaki N, Tadamura E, et al. Age and gender differences in normal myocardial adrenergic neuronal function evaluated by iodine-123-MIBG imaging. J Nucl Med. 1995;36:969-974.

40. Jacobson AF, Lombard J, Banerjee G, et al. ${ }^{123}$ I-MIBG scintigraphy to predict risk for adverse cardiac outcomes in heart failure patients: design of two prospective multicenter international trials. J Nucl Cardiol. 2009;16:113-121. 